\title{
Characterizing parking systems from sensors data through a data-driven approach
}

\author{
Jamie Arjona ${ }^{\mathrm{a},{ }^{*}}, \mathrm{M}^{\mathrm{a} P a z}$ Linares $^{\mathrm{a}}$, Josep Casanovas-Garcia ${ }^{\mathrm{a}, \mathrm{b}}$ \\ ${ }^{a}$ Universitat Politècnica de Catalunya, Barcelona 08034, Spain \\ ${ }^{b}$ Barcelona Supercomputing Center, Barcelona 08034, Spain
}

\begin{abstract}
Nowadays, urban traffic affects the quality of life in cities as the problem becomes ever more exacerbated by parking issues: congestion increases due to drivers searching slots to park. An Internet of Things approach permits drivers to know the parking availability in real time. This research focuses on studying the data generated by parking systems to develop predictive models that generate forecasted information. This can be useful in improving the management of parking areas, while having an important effect on traffic. This work begins by describing the state-of-the-art parking predictive models. Then, introduces the recurrent neural network methods that were used, Long Short-Term Memory and Gated Recurrent Unit, as well as the models developed according to real scenarios in Wattens and Los Angeles. To improve the quality of the models, exogenous variables related with weather and calendar are considered. Finally, the results are described, followed by suggestions for future research.
\end{abstract}

Keywords: parking availability forecast; deep learning; smart cities, recurrent models, time series.

\section{Introduction}

Currently, one of the most important problems in urban areas concerns traffic congestion. This, in turn, has an impact on the economy, nature, human health, cities architecture, and many other facets of life. Part of the vehicular traffic in cities is caused by parking space availability. The drivers of private vehicles usually want to leave their vehicle as close as possible to their destination. However, the parking slots are limited and may not be enough to sustain the demand, especially when the destination pertains to an attractive area. Thus, individuals looking for a place to park their vehicle contribute to increasing traffic flow density on roads where the parking demand cannot be satisfied.

This motivates the use of real-time parking availability in order to build a mathematical model that helps improve parking management. Some approaches to this can be found in (Caicedo et al., 2012) and in (Teodorovic and Lucic, 2006), who use models to manage indoor parking reservations while taking into account future parking demand. However, the nature of the data remains a problem: It is not on-street parking data and is not in real time.

An Internet of Things (IoT) approach allows us to know the state of the parking system (availability of the parking slots) in real time through wireless networks of sensor devices. An intelligent treatment of these data could generate forecasted information that may be useful in improving management of on-street parking, thus having a notable effect on urban traffic. Smart parking systems first appeared in 2015, with platforms in Santander, San Francisco and Melbourne as is explained in (Lin et al., 2017), when those cities began to provide on-street real-time parking data for offering new services to their citizens.

One of the most interesting services is parking availability forecasting, for which the first works studied the temporal and spatial correlations of parking occupancy. For this purpose, a VARIMA model was proposed by Rajabioun and Ioannou (2015) for short-term forecasts (less than 30 minutes) without loss of accuracy.

\footnotetext{
* Corresponding author. Tel.: +34934011019

E-mail address: jamie.arjona@upc.edu
} 
Those short-term forecasts are not useful at all to the end user of this service; thus, prediction intervals on the order of hours are needed. This is similar to what happens in traffic forecasting, where the literature (Vlahogianni and Karlaftis, 2011) identified that the processes that occur on traffic are of long-term nature with high volatility. In order to overcome these problems, Vlahogianni and Karlaftis (2013) propose a potential class of models that can offer better predictions than the commonly used ARIMA models: Neural Networks (NN).

The purpose of this paper is to present models that accurately forecast parking availability in urban areas in order to create and improve services for parking management. These models are developed for real scenarios using data provided by sensors with certain inaccuracies due to their very nature. Furthermore, the developed models are based on Recurrent Neural Networks (RNN), as these can learn the time-dependency component that occurs in parking availability data. Lastly, the forecast will be of a long-term duration, considering an interval of time between 1 and 6 hours.

This paper is organized as follows. The next section presents a summarized literature review of forecasting methods related to smart cities, specifically within the Intelligent Transportation Systems (ITS) area. Based on the current state of the art and the problem at hand, two methods are then proposed. Following, based on the type of data available, the proposed architectures are described. Next, data from two different scenarios are analyzed in order to take decisions on the modeling. Then, validation tests are presented on the developed models and a comparison is made with one of the most relevant methods proposed recently in the literature. The last section discusses the empirical findings from the implemented scenarios, followed by some concluding remarks.

\section{State of the Art}

In recent years, the research on parking forecasting has grown more sophisticated due to the availability of realtime data on parking slots. These data have become available as a result of advances in the field of sensors and the socalled IoT paradigm. The usual methods for obtaining parking data are sensors, VANET (vehicle ad-hoc network), and GPS for detecting the number of vehicles in a zone's cruising traffic. The variables of interest are availability, reservation (Caicedo et al., (2012)) and occupancy (usually in the form of parking occupancy rate (OCCR) (Vlahogianni et al., (2014)).

Prediction for parking analytics has always been a driving force behind traffic predictive analytics, which has been an active area of research for many years. This in turn has motivated efforts to adapt promising traffic methods to parking prediction. Thus, this section considers the scope of both in the literature review.

One of the first works that used an NN model is (Ishak et al., (2003), where upstream and downstream data from a target link are used for traffic flow prediction. In (Vlahogianni et al., 2005), a Multilayer Perceptron approach with a Genetic Algorithm is used for meta-optimization (or hyper-parameter tuning) in the short-term forecasting of traffic flow. At the same time, Medeiros et al. (2006) presented a work that they called AR-NN, where an NN is modeled using a statistical approach like the ones used in time series.

Currently, the approaches are centered on Deep Learning (large NN models with more than one hidden layer). In (Yanjie Duan et al., 2016), Long Short-Term Memory NN (LSTM) (Hochreiter and Schmidhuber, 1997)) are used for training 66 models (one per link) in order to perform travel flow forecasting. Fu et al. (2017) presented a comparison between ARIMA, LSTM and Gated Recurrent Unit (GRU) (Cho et al., (2014)) for travel flow. Another approach with a Deep NN is presented in (Polson and Sokolov, 2017), where the model is used for traffic flow forecasting.

As mentioned in the Introduction, the first works on parking prediction emerged in parallel with big IoT platforms that collected data in real time from parking spots. (Vlahogianni et al., 2016) takes an NN approach to predict parking occupancy rate by using Time Delay Neural Network models. Blythe et al. (2015) propose transforming parking space availability time series in order to reconstruct them in phase space, and then use the transformed data to train what they call a Wavelet NN.

Naïve Bayes and a decision tree are used in (Hsueh et al., 2018) for predicting parking occupancy as a categorical variable (level: low, mid, high) at arrival time. The relevance of the study is that it uses three sets of different types of features: temporal (day of the week, time of day (ToD) and occupancy rate); spatial (nearest parking sectors, similar parking sectors based on RMSE, similar parking sectors based on RMSE with different ToD); and others (sector id and hourly precipitation). 
Another approach to the parking prediction problem is detecting traffic that results from cruising. In (Jones et al., 2018), data from smartphones (GPS, accelerometer, magnetometer, and gyroscope) are used to detect cruising behavior. The detection of cruising can be useful for updating probabilistic parking availability in the desired zone. Using GPS data, map matching is carried out via a Hidden Markov Model to localize the zones where the vehicle is located. Cruising is detected as a significant local minimum in the GPS trace relative to the distance to the destination. Data from the other sensors are used for training the ML algorithms. The ML algorithms that ascertain whether or not the user is cruising are Decision Tree, K-NN, and SVM. One of the problems with this proposal is that accuracy depends on the number of users in the zone.

In this work, our objective is to take an RNN approach to forecast the occupancy of street parking sectors over a succeeding period of 6 hours. We used LSTM and GRU models, as they have been proven to be the best RNN architectures for solving a wide set of sequential data problems (Jozefowicz and Zaremba, 2015). As far as we know, this approach has been used recently with good results in traffic forecasting but not for parking. In addition, these kinds of models allow improving predictions via the use of exogenous data such as weather conditions and calendar effects (holidays, day of the week, month, etc.). The reason this is interesting is that sensor measurements are subject to environmental noise that can affect the quality of the data used for training the models. Thus, relying only on measurements cannot be enough.

\section{Theoretical issues about the proposed models}

\subsection{Methods}

RNNs are part of the NN framework and are specialized for working with sequential data. The computational units are connected to themselves in order to preserve past information that can be relevant in the present computation. An RNN is composed of a weight matrix $W_{x}$ that is applied to the input, a hidden parametric state transition $S$, a weight matrix $W_{y}$ that is applied to the output and an output bias $b_{y}$. The input consists of a sequence of $T$ elements $x=$ $\left(x_{1}, \ldots, x_{T}\right)$. At each step $t$, the RNN unit computes a hidden state $s_{t}=S\left(s_{t-1}, x_{t}\right)$ that is part of the hidden sequence $s=\left(s_{1}, \ldots, s_{T}\right)$, and an output $y_{t}=W_{y} s_{t}+b_{y}$ that is part of the output sequence $y=\left(y_{1}, \ldots, y_{T}\right)$.

There exist different implementations of RNN and, as mentioned in the Section 2, two of them are well-known in the literature, namely LSTM and GRU. These architectures make use of auxiliary mechanisms called gates, which enhance the computational units in order to manage past information better than classic RNN units allowing the models to have longer memory and to be more robust to problems that arise in RNN, namely the vanishing and exploding gradients (detailed information is given in the work (Hochreiter, 1991)).

Specifically, LSTM stores memory in the form of an internal state similarly to RNN and also implements a gating system that modulates the flow of information within the unit in order to handle the interaction between the state and the current sample. This flow of information is known as the cell state, where the information is added and removed carefully by a structure of gates that are composed of a sigmoid function and a pointwise multiplication operation. An LSTM has four of these gates, which control the hidden state of the LSTM neuron in the following manner. First, the forget gate is computed in order to ascertain the relationship between the current input and the hidden state, this will be used in order to remove parts of the cell state that are no longer needed. Then, the update gate is computed in order to decide if the input is relevant or not to add it to the flow of information. With the results, the internal state gate is computed based on how much relevant previous information and new information is retained. Finally, the output of the neuron is computed by considering the new internal state.

The difference between LSTM and GRU basically is that a single gate controls the forgetting and the decision to update the state, thus requiring less computation. It is interesting to note that GRU models have not been used before in the literature for parking occupancy rate forecasting. In addition, the use of NN permits to output a vector of predictions that behave like different steps leading up to forecasts.

Intuitively, these models seem to be perfect candidates for learning temporal behaviours about time series data as they can learn linear and nonlinear patterns subject to time. Another advantage of these models is the easy integration of other information sources (exogenous variables) that can have an effect on the forecasts. 


\subsection{Input Data}

Data used in this work are formed by data from parking sensors and data from weather and calendar sources of information, obtained from publicly available APIs. The data from the parking sensors is called the endogenous data because past realizations are used in order to predict future outcomes of itself. The other are called exogenous data as they do not belong to the parking process but can contribute or have an influence over it. Because the availability of parking data is scarce, it is important to know the minimum amount of data needed to train the models. This has brought us to try RNN models in other scenarios where more historical data is available.

\subsubsection{Parking Data}

This research takes advantage of the availability of real-time parking data provided by Worldsensing S.L. company (https://www.worldsensing.com/product/fastprk/), specialists in the manufacturing of sensors devices for cities and industries. Their sensors are buried in the road within the area delimited by the parking spot and they are event-based. When they detect a car over them, a change in the sensor measurements happens indicating that the parking spot has been occupied. The data are sent to a gateway and from there to servers in the Cloud. Sensors in the same proximity zone send their data to a unique gateway; this characteristic is what defines a sector (Worldsensing S.L. convention). Thus, these input data can be handled at different scales: parking spot (sensor level) and sector. The following specifies each of these measures:

- Sensor measurements are event-based and indicate when a parking spot is occupied and when it is free. They are indexed by timestamp and contain information on each sensor event at the time that it was generated. These data are collected from the gateways.

- Sector measurements contain information related to the sector status at each hour of the day. Sector data are computed by an extract-transform-load (ETL) process that aggregates the status of the sensors in each sector. These are the data that has been used for this study.

The developed forecasting models predict the occupancy rate by hour for a sector. This is computed by aggregating the occupancy/hour for all the sensors in the same sector. Occupancy/hour is a measurement between 0 and 1 , where 0 refers to an empty sector for an hour and 1 refers to full occupancy.

\subsubsection{Exogenous data}

Because parking data may not be enough due to parking availability being affected by other sources of information, this study uses calendar effects and weather.

- Calendar effects: Parking behavior can be affected by so-called calendar effects. Occupancy on a weekend is different than on weekdays, but the pattern is maintained over weekends. In this study, the following calendar information is considered: day of the week, season, weekday and holiday (global and local). These data was collected from https://calendarific.com/api/v2/

- Weather: The objective of using this source of information is to find if the parking occupancy patterns are modified by the weather. The information considered related to weather is temperature, wind speed, precipitation intensity and weather summary for each hour. These data were gathered from https://api.darksky.net/forecast/.

It is worth mentioning that some of these data are categorical and cannot be used directly as input of a NN model. In order to use it, it is needed to do a preprocessing of the data and the use of a special kind of layer (details are given in Section 3.3).

\subsubsection{Training Data}

The proposed methodology begins by studying the amount of historical data needed for training models that make better predictions than random guesses. This quantification uses more than one year of historical data from different parking sensors deployed in Sandvika (Norway), Castelfranco Veneto and San Doná di Piave (Italy). These studies 
show that more than three months' worth of data is enough to enable RNN models to learn the occupancy patterns that occur in parking sectors. As more data become available, the forecasts are improved (see Figure 1) until arrive to the irreducible noise. Thus, the proposed work uses seven months of training data for the different city parking sites in order to compare the two previously selected RNN methods: LSTM and GRU. An extra month of data is used to test the accuracy.

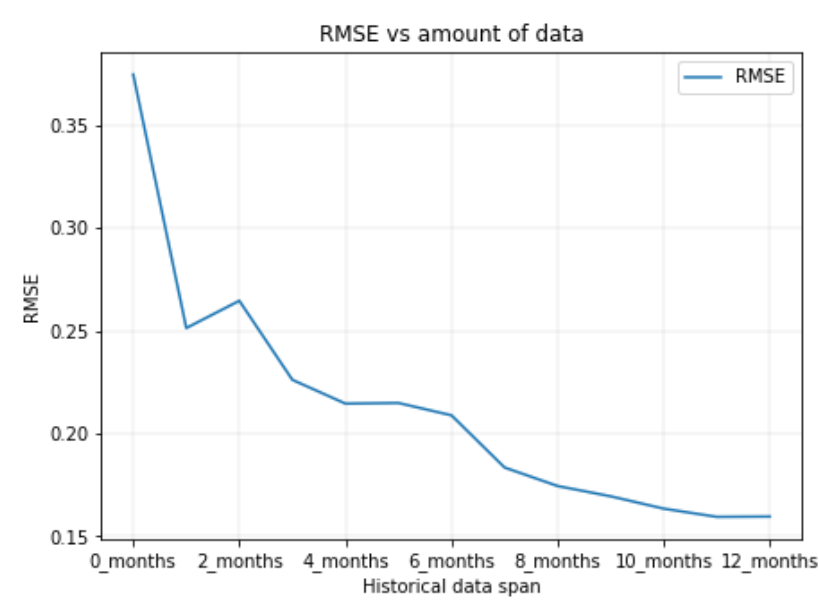

Figure 1 Root Mean Squared Error on data availability, by months.

\subsection{Models Architecture}

With the aim of finding the best architecture different combinations of the proposed variables are considered in order to detect the ones that contribute more to improve the accuracy.

First, a model using only the endogenous variable is used as a baseline. This endogenous model uses as an input sequences of $\tau$ elements where the last element is the parking occupancy at instant time step $t$ and the first element is for the time step $t-\tau$. These sequences of fixed window of $\tau$ elements form a matrix where each row is obtained rolling the window over the corresponding amount hours for each data set. A second matrix is used as the output where each row is a sequence of 6 elements as those elements are the parking occupancy for the time steps $t+1$ to $t+6$ and are used to train the model and to evaluate its results at the test stage. These data are used as input of the recurrent neural networks (LSTM or GRU) layers. Figure 2 shows the proposed architecture for the endogenous model.

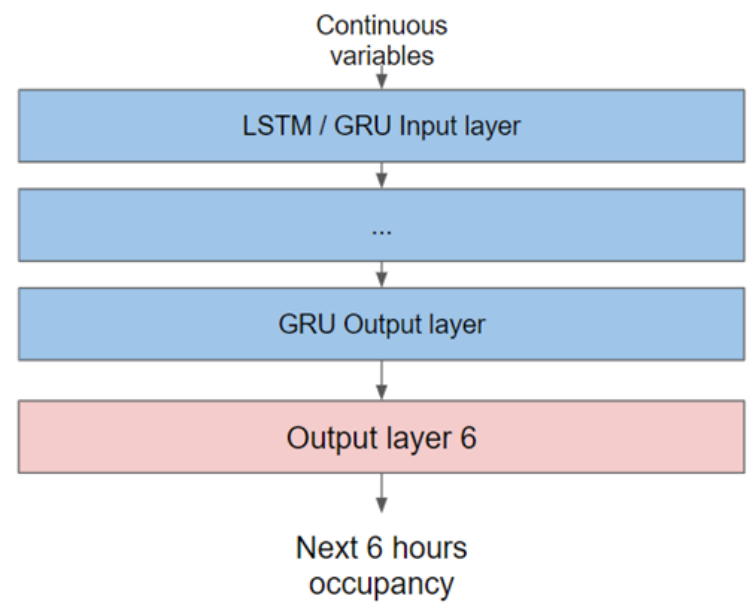


Figure 2 Endogenous model architecture.

On the other hand, in order to use all the variables, it is proposed an architecture of the model where recurrent neural networks layers are used for the numerical variables as they are organized with a rolling window approach like in the endogenous model. For the categorical variables, one embedding layer is used for each of them. The categorical variables are used to form embeddings, that are projections of the discrete categories into a continuous vector space. Embeddings are learnt during training as their values depends on the parameters of the model. In (Guo and Berkhahn, 2016) a similar idea was pursued. The embeddings outputs are concatenated with the output of the recurrent neural network layers part of the model forming vectors that represent learned features from the input data. The new representations of the input data are then used to feed two dense layers of 128 units each, this approach is used by well-known convolutional NN models. Lastly, the model has an output layer of six units, one for each of the forecasted hours. The use of these six units allows that the model learns the patterns at different time steps of predictions and returns a vector of length six. In methods like ARIMA this cannot be done as the output consists in a single value, in order to give consecutive predictions, a multistep strategy is used, where the prediction is used as input in order to generate the following prediction. Figure 3 shows the proposed architecture if all the exogenous variables (numerical and categorical) are used with the endogenous one.

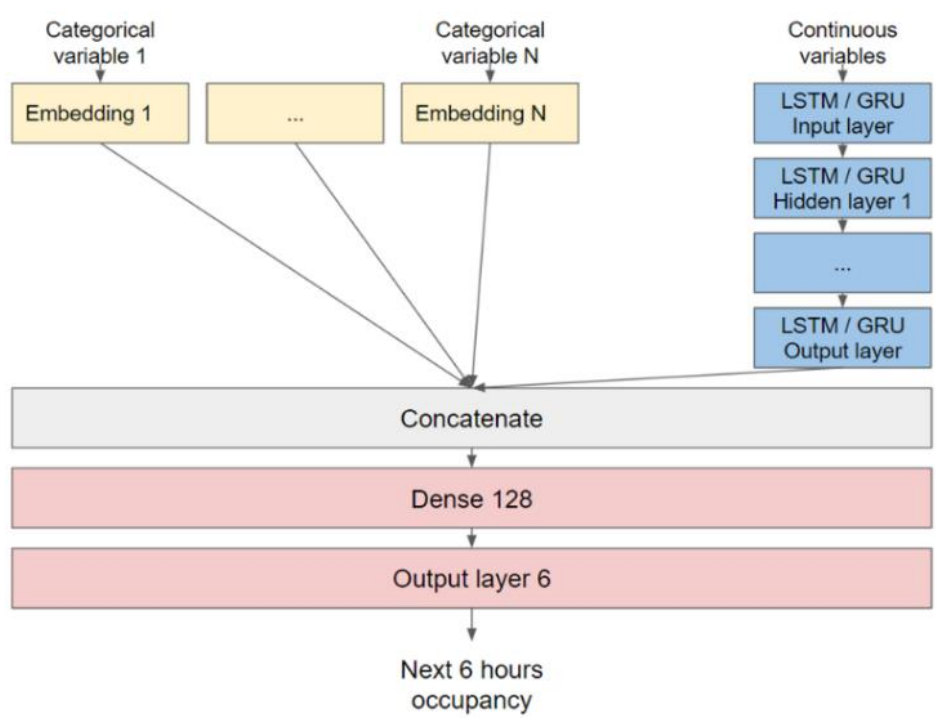

Figure 3 Proposed model architecture including all the variables.

Above has been presented the general architecture of the models in order to be able to make use of the data at hand. There are other aspects, some of them part of the architecture and others from the NN methodology, that cannot be decided beforehand. These other aspects are known as hyperparameters as their value cannot be optimized together with the optimization of the parameters of the model. The hyperparameters that affect the architecture of the model are the number of layers, the number of neurons for each of the hidden layers, the activation function of the neurons (which we considered linear, ReLu, SeLu, sigmoid and the hyperbolic tangent) and dropout rate (a technique for regularization, explained in (Srivastava et al., 2014)). Other hyperparameters that affects the model are the optimizer method, its parameters and the method used in order to give initial values to the parameters of the model.

In this work, a random search strategy (Bergstra and Bengio, 2012)) has been considered in order to select the value of those hyperparameters related to the architecture of the model. For the other hyperparameters, the most common values from the literature has been used, those are: Adam as optimizer, with learning rate of 0.001 and $\beta_{1}$ and $\beta_{2}$ with values 0.9 and 0.999 respectively (Kingma and Lei Ba, 2014); Glorot, as a method for initializing the weights (Glorot 
and Bengio, 2010). An early stopping criterion proposed in (Caruana and Lawrence, 2000) is employed in the random search in order to quickly discard models with poor initial results while training saving computation and time.

\section{Scenario Data Analysis}

\subsection{Scenarios}

Some of the data used in this work comes from a pilot program carried out as part of the Horizon 2020 project titled Fastprk2: Enhanced on-street parking management system (https://cordis.europa.eu/project/rcn/204264/factsheet/en) which included the cities of Antwerp, Wattens, Paris, Grudziadz and Los Angeles.

Specifically, this work makes use of the data collected from parking sectors in the cities of Wattens (Austria) and Los Angeles (USA). These two scenarios have been chosen because their sectors present unique characteristics like indoor/outdoor, public/private, payment/free and parking end user.

Table 1 shows the sectors of both scenarios and their number of sensors. Figure 4 shows their location.

\begin{tabular}{cccc}
\multicolumn{3}{c}{ Table 1 Sectors characterization for Wattens and Los Angeles scenarios. } \\
\hline Scenario & Sector name & Number of sensors & Deployment date \\
\hline \multirow{2}{*}{ Wattens } & Inside & 22 & $02 / 03 / 2018$ \\
& Outside & 18 & $02 / 03 / 2018$ \\
Los Angeles & Standard & 60 & $01 / 04 / 2018$ \\
& Permit & 31 & $01 / 04 / 2018$ \\
& Disabled & 5 & $01 / 04 / 2018$ \\
& ZipCar & 4 & $01 / 04 / 2018$ \\
\hline
\end{tabular}

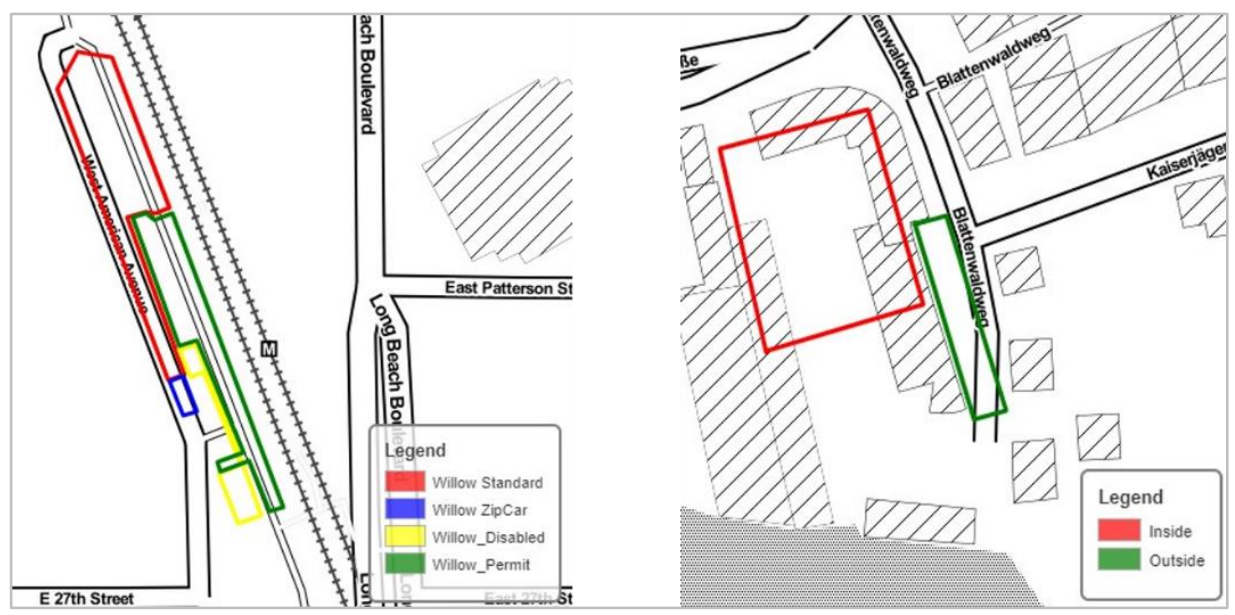

Figure 4 Scenarios maps. Left are Los Angeles sectors, right are Wattens sectors.

It is important to note that the Wattens sectors are private parking places used by the employees of the SWARCO company while the sectors of the Los Angeles Willow zone are used for Park and Ride as they are next to the metro rail station and they have different pay-per-use restrictions. This heterogeneity makes impossible to work with a single model for all the sectors in a city, so a model has been developed for each of the sectors in each city. This study presents the results for the sectors where more sensors are involved. It is also important to note here that these parking 
sectors were deployed in the year 2018, therefore the historical data available cover April 1st to November 30th, 2018. November data have been used as the test set in order to evaluate the models with unseen data.

\subsection{Data analysis}

A visual exploratory analysis of the occupancy/hour data for each of the sectors has been performed in order to reveal information that could help in the development of the models and characterize the sectors. This analysis includes:

- Tables containing information about the sectors like its name, number of sensors (that can be considered parking spots) and deployment date.

- Map of the localization of the sectors.

- Visualizations of monthly data by choosing a random month.

- In order to check if the visualized monthly patterns are repeated through all the data available, seasonal plots are used. In these plots the occupancy/hour data are averaged for all the days of the week.

- Box plots have been used in order to check changes in the levels of the variance and the mean through the time.

- Plots of the autocorrelation and partial autocorrelation functions (ACF and PACF) in order to detect linear dependencies between data realizations and past realizations.

- Cross-correlation visualizations that could help in order to detect spatiotemporal linear dependencies.

Next, the most relevant findings are presented for the sectors with the largest number of sensors.

\subsection{Visualizations}

Data realizations for the month of October 2018 are shown in Figure 5 for the Wattens sector Inside, and Figure 6 for Los Angeles sector Standard. Observe that both sectors present common patterns that show how the parking occupancy process is greatly influenced by the working days. Clearly, the levels of occupancy are different in the weekdays when comparing to the ones from the weekends. Also, notice that the parking occupancy levels on holidays are lower when compared to non- holidays days. This can be noticed in Figure 5, where Friday 26 of October has lower levels of occupancy as it coincides with the Austrian National Day. It is interesting to note that in the Wattens sector, the daily patterns go along with office work that are 8 am to $17 \mathrm{pm}$ from Monday to Thursday and 8 am to 12 pm on Friday while in the Los Angeles sector, occupancy levels reach its peak at 6 am and start to decrease after 18 pm, mainly influenced by the fact that the sector is next to a metro rail station. 


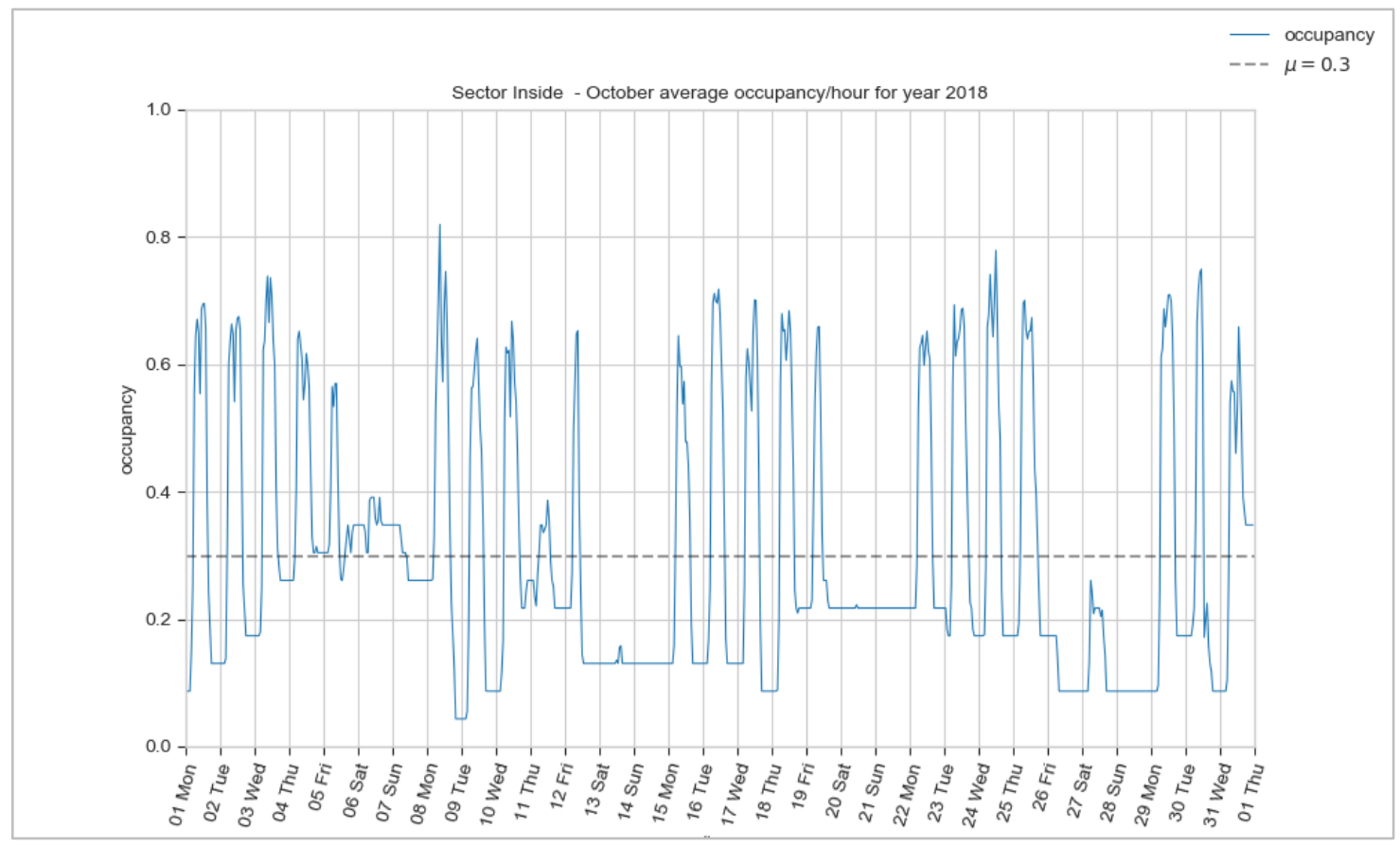

Figure 5 Wattens Inside sector time series for October 2018.

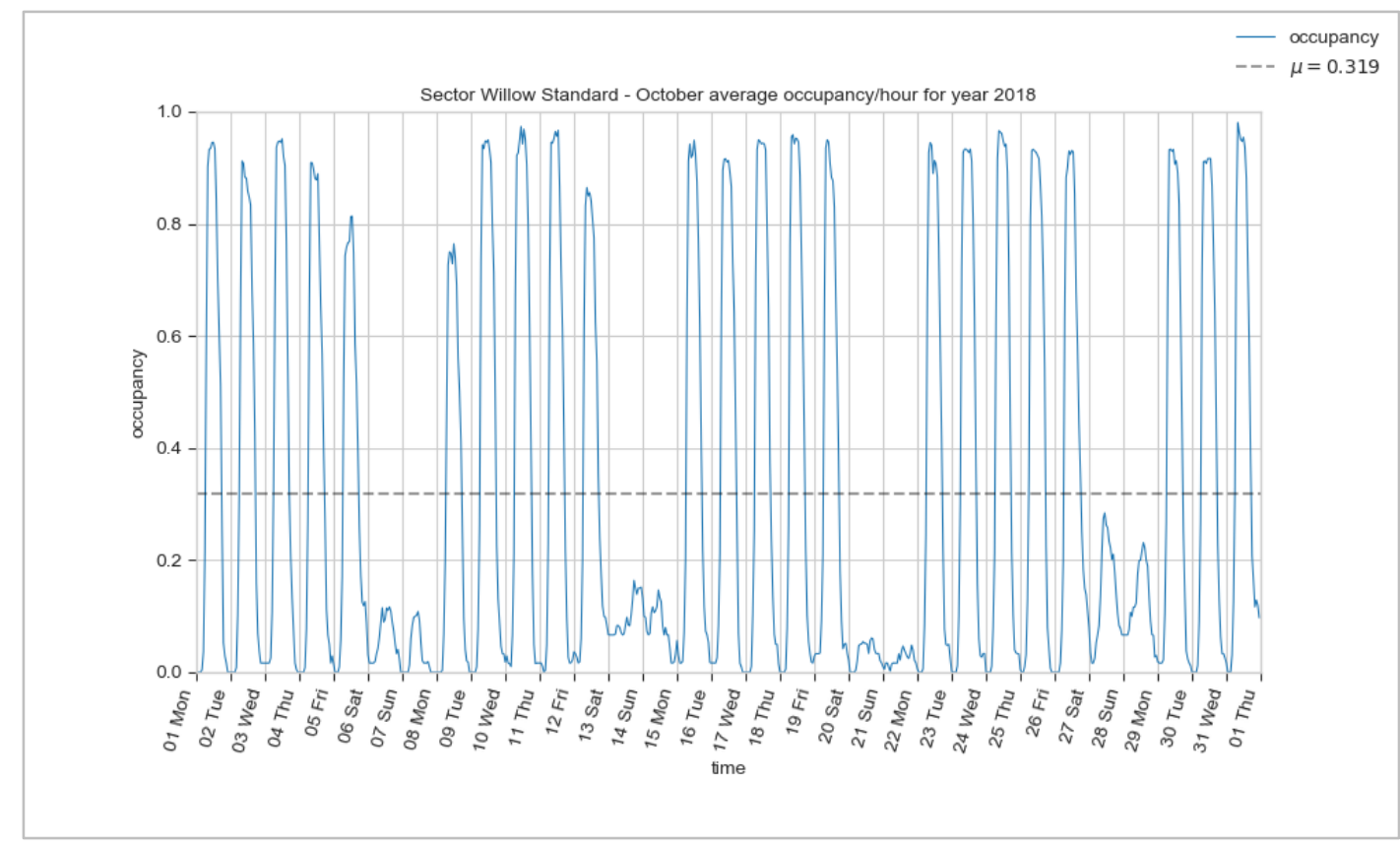

Figure 6 Los Angeles Standard sector time series for October 2018.

In order to check if the presented patterns for October are repeated through all the data available, a seasonal plot is used. The seasonal plot shows the average occupancy for each hour of the day and for every day of the week. If it presents a similar pattern through the days as in the monthly plots (like the above plots, where hourly occupancy levels for the month of October are shown), then it can be assumed that data behave similar for the time span of data available. 
Figure 7 presents both sectors seasonal plots, visually confirming that October patterns are also repeated through all the data used. This work assumes that the presented behaviours of the occupancy levels from each of the sectors persist in time.

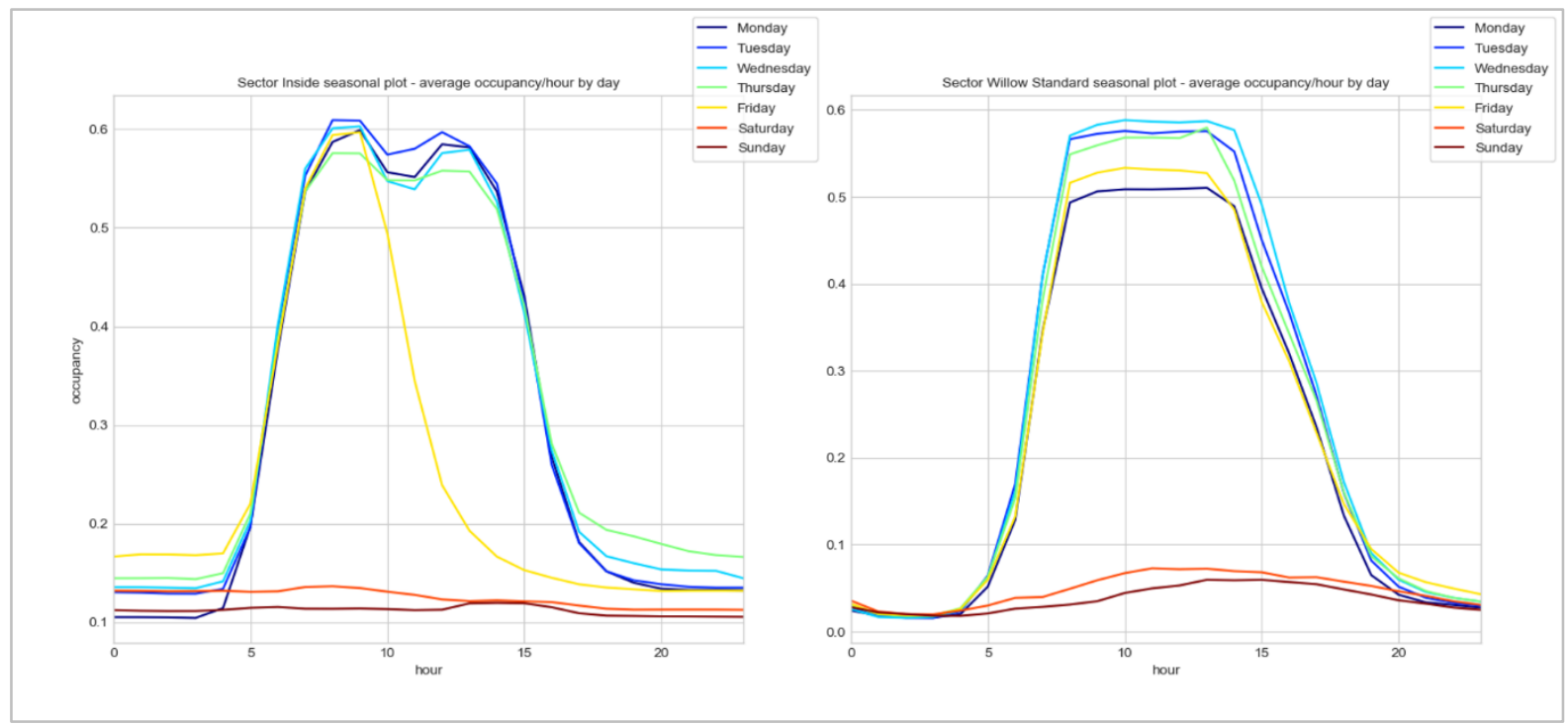

Figure 7 Seasonal daily plot for Wattens Inside (left) and Los Angeles Standard (right) sectors for the year 2018.

Box plots reveals if the values of the variance and the mean have been changing through the interval of data available. This is of special importance in time series modeling problems as it is needed that the mean and variance values remain constant through all the data. This property of the data is known as stationarity. Figure 8 shows the box plots for all the data available of sectors Wattens Inside and Los Angeles Standard. Both box plots present that the mean and the variance have remained similar for all the interval of time considered.
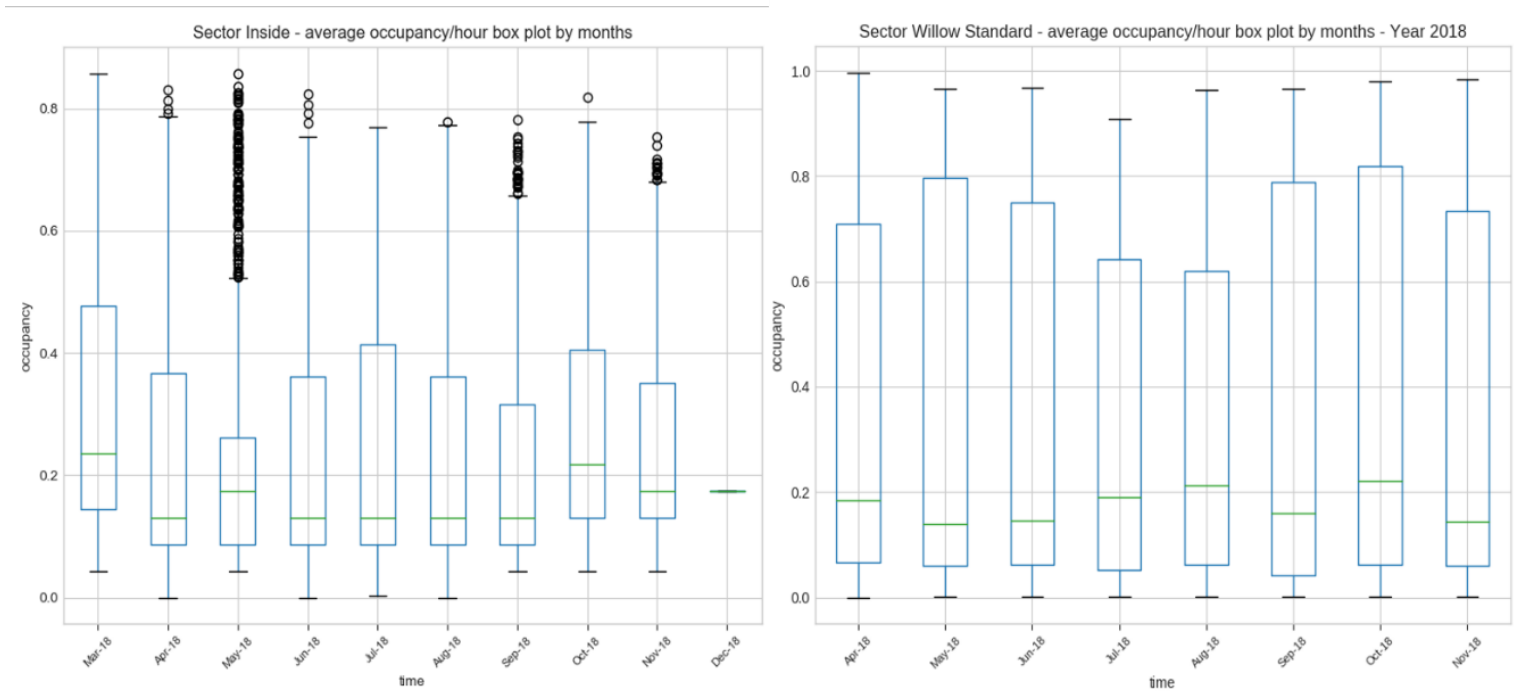

Figure 8 Boxplots for Wattens Inside (left) and Los Angeles Standard (right) sectors for the year 2018. 
The ACF and PACF plots of the October month for both sectors are presented in Figure 9 and help to visualize the linear dependency (correlation and partial correlation) through different time lags. It can be noticed that the most important lags are the first two and the lags twenty-four and twenty-five, indicating daily seasonality. This fact has been used in order to divide the data in sequences of 24 elements that will be used as input of the recurrent layers as mentioned in Section 3.3.

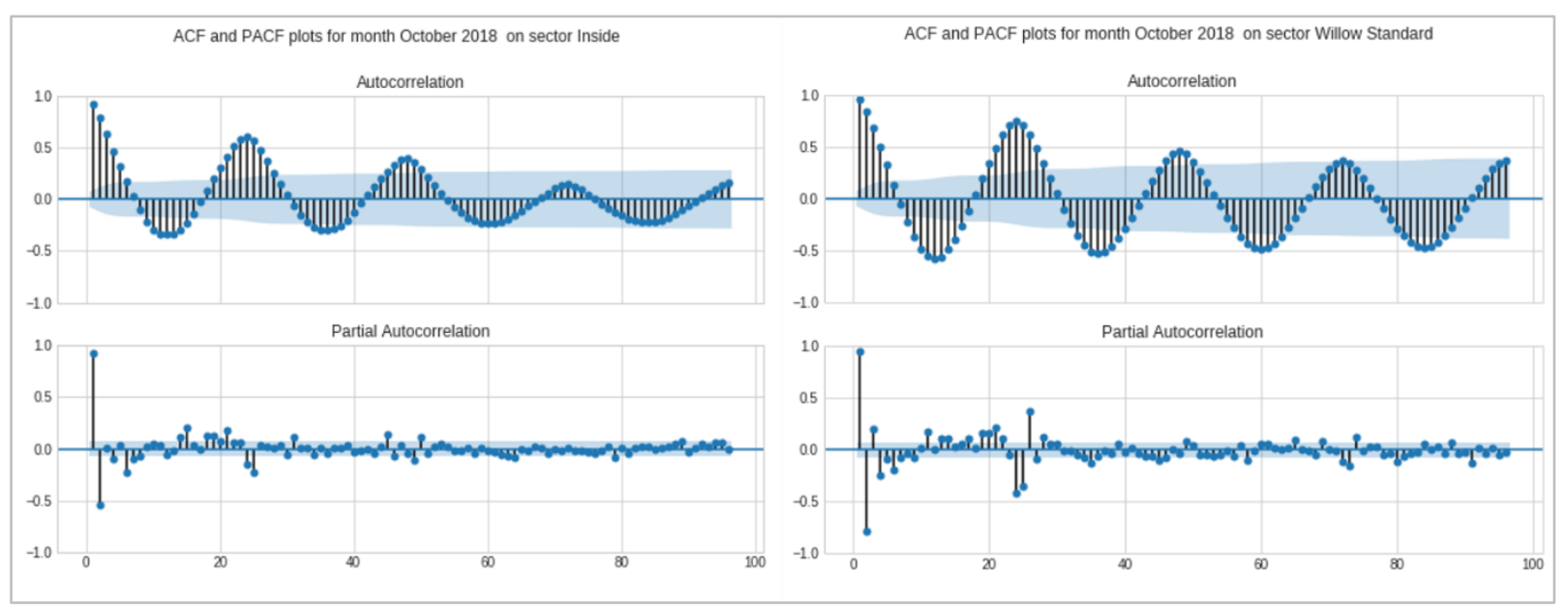

Figure 9 ACF and PACF plots for Wattens Inside (left) and Los Angeles Standard (right) sectors for October 2018.

\section{Results and discussion}

Models are evaluated and compared using the Root Mean Square Error metric (RMSE), which is a commonly used metric for model goodness of fit and comparison. This study presents only the occupancy forecasting results of the sector with the most sensors in each of the cities, that are, the sector Inside for Wattens and the sector Standard for Los Angeles. Table 2 shows the comparison of the different models using the architectures proposed, LSTM and GRU, with models from the Time Delay Neural Network (TDNN) architecture, as it was the one proposed and used in the relevant work (Vlahogianni et al., 2016). TDNN are part of the NN family of models, with one hidden layer and one output layer that lacks the recurrent connection that architectures like the ones proposed have, making them unable to share parameters across the time dimension. The RMSE obtained with the endogenous model (parking occupancy) is compared with the model that uses parking occupancy and all the exogenous variables (weather and calendar).

Table 2 Error comparison (RMSE) of the proposed architectures.

\begin{tabular}{|c|c|c|c|c|c|c|c|c|}
\hline \multirow{2}{*}{ City } & \multirow{2}{*}{ Method } & \multirow{2}{*}{ Architecture } & \multicolumn{6}{|c|}{ Error accuracy (RMSE) } \\
\hline & & & 1 hour & 2 hours & 3 hours & 4 hours & 5 hours & 6 hours \\
\hline \multirow{6}{*}{$\begin{array}{r}\text { Los } \\
\text { Angeles }\end{array}$} & \multirow{2}{*}{ TDNN } & Endogenous & 0.07985 & 0.09953 & 0.12113 & 0.13755 & 0.16091 & 0.17573 \\
\hline & & Parking + Exogen. & 0.12484 & 0.14494 & 0.17587 & 0.19435 & 0.22006 & 0.22909 \\
\hline & \multirow{2}{*}{ LSTM } & Endogenous & 0.04834 & 0.06900 & 0.10552 & 0.13379 & 0.15521 & 0.17175 \\
\hline & & Parking + Exogen. & 0.07152 & 0.08764 & 0.12052 & 0.14376 & 0.16855 & 0.18427 \\
\hline & \multirow{2}{*}{ GRU } & Endogenous & 0.04532 & 0.07393 & 0.10471 & 0.13237 & 0.15407 & 0.17144 \\
\hline & & Parking + Exogen. & 0.06598 & 0.09442 & 0.11940 & 0.13945 & 0.15891 & 0.17799 \\
\hline \multirow{6}{*}{ Wattens } & & Endogenous & 0.05518 & 0.07824 & 0.09407 & 0.10832 & 0.11689 & 0.12154 \\
\hline & TDNN & Parking + Exogen. & 0.06180 & 0.08945 & 0.10312 & 0.11479 & 0.12515 & 0.13080 \\
\hline & \multirow{2}{*}{ LSTM } & Endogenous & 0.04671 & 0.06959 & 0.08612 & 0.09946 & 0.10787 & 0.11270 \\
\hline & & Parking + Exogen. & 0.05834 & 0.09380 & 0.10346 & 0.11545 & 0.11967 & 0.12732 \\
\hline & \multirow{2}{*}{ GRU } & Endogenous & 0.04364 & 0.06913 & $\mathbf{0 . 0 8 5 7 7}$ & 0.09864 & 0.10627 & 0.11147 \\
\hline & & Parking + Exogen. & 0.05155 & 0.08317 & 0.10726 & 0.10591 & 0.10540 & 0.11504 \\
\hline
\end{tabular}


In Los Angeles sector, the best obtained RMSE is from 4.5\% to 17\% (from 1-hour to 6-hours forecasted, respectively) and for the Wattens sector is from $4.4 \%$ to $11.1 \%$. Both methods (LSTM and GRU) performed similar if considering only the endogenous variables (although the differences are of less than $0.3 \%$ ) and outperformed the TDNN models. TDNN models with the endogenous variables present some competitive results if compared with GRU and LSTM, but they achieve worse error when exogenous variables are used. In addition, the results show that in all the cases the endogenous models obtain better results than the ones with all the exogenous variables. This appears to show that not all the exogenous variables help in the improvement of the quality of the results. In order to quantify the effect of the variables, new experiments have been made with the LSTM and GRU architectures.

Table 3 and Table 4 detail for Wattens and Los Angeles, respectively, presents the obtained results when the parking variable is used in combination with each of the exogenous variables, independently. In particular, they illustrate that not all the exogenous variables do improve the predictions. In the case of the calendar variables (day of week, holiday, working day, month and season) only the day of the week and the working day information play a part in the improvement of the accuracy. The former shows a significant increase in the accuracy of the GRU models (average improvement of $1.5 \%$ ) and the latter improves all the experiments (average improvement of $0.5 \%$ ).

Table 3 Results with each of the exogenous variables for the parking sectors in Wattens.

\begin{tabular}{cccccccc}
\hline \multirow{2}{*}{ Method } & Architecture & \multicolumn{5}{c}{ Error accuracy (RMSE) } \\
& Parking + Exogenous & 1 hour & 2 hours & 3 hours & 4 hours & 5 hours & 6 hours \\
\hline & Day of week & $\mathbf{0 . 0 4 5 5 3}$ & $\mathbf{0 . 0 6 5 4 4}$ & $\mathbf{0 . 0 7 6 9 7}$ & $\mathbf{0 . 0 8 4 0 2}$ & $\mathbf{0 . 0 8 4 9 2}$ & $\mathbf{0 . 0 8 2 4 1}$ \\
& Holiday & 0.04559 & 0.07006 & 0.08744 & 0.10221 & 0.11206 & 0.11853 \\
& Working day & 0.05009 & 0.07057 & 0.08271 & 0.09138 & 0.09335 & 0.09125 \\
LSTM & Month & 0.06237 & 0.07964 & 0.10111 & 0.10985 & 0.12973 & 0.11569 \\
& Season & 0.05097 & 0.07128 & 0.08733 & 0.10074 & 0.10878 & 0.11340 \\
& Precipitation intensity & 0.04568 & 0.06968 & 0.08655 & 0.09996 & 0.10788 & 0.11233 \\
& Weather summary & 0.04789 & 0.07170 & 0.08770 & 0.10139 & 0.10966 & 0.11470 \\
& Temperature & 0.07154 & 0.08910 & 0.10064 & 0.11002 & 0.11600 & 0.11919 \\
& Wind speed & 0.04915 & 0.07201 & 0.08744 & 0.09882 & 0.10560 & 0.10960 \\
& & & & & & \\
& Day of week & $\mathbf{0 . 0 4 2 0 7}$ & $\mathbf{0 . 0 6 3 7 2}$ & $\mathbf{0 . 0 7 6 0 7}$ & $\mathbf{0 . 0 8 4 1 7}$ & $\mathbf{0 . 0 8 4 9 1}$ & $\mathbf{0 . 0 8 3 8 2}$ \\
& Holiday & 0.04597 & 0.07026 & 0.08707 & 0.10046 & 0.10955 & 0.11715 \\
& Working day & 0.04401 & 0.06723 & 0.08035 & 0.09026 & 0.09279 & 0.09212 \\
GRU & Month & 0.04813 & 0.06997 & 0.08496 & 0.09976 & 0.10807 & 0.11339 \\
& Season & 0.04357 & 0.06869 & 0.08522 & 0.09790 & 0.10586 & 0.11184 \\
& Precipitation intensity & 0.04363 & 0.06931 & 0.08645 & 0.09974 & 0.10739 & 0.11250 \\
& Weather summary & 0.04323 & 0.06817 & 0.08414 & 0.09846 & 0.10633 & 0.11217 \\
& Temperature & 0.04911 & 0.07813 & 0.09683 & 0.10911 & 0.11427 & 0.11677 \\
& Wind speed & 0.04566 & 0.07077 & 0.08738 & 0.09952 & 0.10667 & 0.11154 \\
\hline & & & & & & &
\end{tabular}

Table 4 Results with each of the exogenous variables for the parking sectors in Los Angeles.

\begin{tabular}{cccccccc}
\hline \multirow{2}{*}{ Method } & Architecture & \multicolumn{5}{c}{ Error accuracy (RMSE) } \\
& Parking + Exogenous & 1 hour & 2 hours & 3 hours & 4 hours & 5 hours & 6 hours \\
\hline \multirow{4}{*}{ LSTM } & Day of week & 0.05811 & 0.07934 & 0.11096 & 0.13464 & 0.14842 & 0.16130 \\
& Holiday & 0.05276 & 0.07093 & 0.10835 & 0.13480 & 0.15288 & 0.16833 \\
& Working day & 0.04982 & 0.06937 & 0.10223 & 0.12621 & 0.14181 & 0.15343 \\
& Month & 0.07912 & 0.10417 & 0.11563 & 0.13632 & 0.16012 & 0.17830 \\
& Season & 0.05463 & 0.07817 & 0.10730 & 0.13295 & 0.15134 & 0.16736 \\
& Precipitation intensity & $\mathbf{0 . 0 4 5 3 5}$ & $\mathbf{0 . 0 6 5 3 6}$ & $\mathbf{0 . 1 0 0 2 1}$ & $\mathbf{0 . 1 2 6 6 0}$ & $\mathbf{0 . 1 4 6 5 7}$ & $\mathbf{0 . 1 6 2 9 1}$ \\
& Weather summary & 0.06867 & 0.08276 & 0.12089 & 0.14666 & 0.16594 & 0.18227 \\
& Temperature & 0.05520 & 0.07599 & 0.11914 & 0.14888 & 0.17076 & 0.18869 \\
& Wind speed & 0.05194 & 0.07319 & 0.10861 & 0.13378 & 0.15307 & 0.17009
\end{tabular}




\begin{tabular}{|c|c|c|c|c|c|c|c|}
\hline \multirow{9}{*}{ GRU } & Day of week & 0.04174 & 0.06626 & 0.09361 & 0.11817 & 0.13587 & 0.14874 \\
\hline & Holiday & 0.04782 & 0.07507 & 0.10561 & 0.13303 & 0.15359 & 0.16999 \\
\hline & Working day & 0.04932 & 0.07623 & 0.10654 & 0.13150 & 0.14873 & 0.16017 \\
\hline & Month & 0.05855 & 0.08636 & 0.10642 & 0.13399 & 0.15595 & 0.18286 \\
\hline & Season & 0.05861 & 0.08026 & 0.11261 & 0.14176 & 0.15957 & 0.17790 \\
\hline & Precipitation intensity & 0.05556 & 0.08909 & 0.12091 & 0.14777 & 0.16992 & 0.18790 \\
\hline & Weather summary & 0.04796 & 0.07503 & 0.10539 & 0.13177 & 0.15364 & 0.17099 \\
\hline & Temperature & 0.04789 & 0.07869 & 0.11263 & 0.14443 & 0.16752 & 0.18451 \\
\hline & Wind speed & 0.04711 & 0.07618 & 0.10906 & 0.13772 & 0.15913 & 0.17620 \\
\hline
\end{tabular}

On the other hand, the month variable presents unexpected results with a negative impact in the LSTM accuracy (average reduction of the accuracy of 1.2\%). Furthermore, the models including season and holidays information show inconclusive results.

In contrast, the weather variables (precipitation intensity, weather summary, temperature and wind speed) do not clearly contribute to the accuracy (RMSE of $\pm 0.5 \%$ ) of the proposed methods for the analyzed parking sectors. In fact, it is important to note that the temperature variable does not improve the predictions for any forecasted hour for any city sector and for any of the proposed methods.

Furthermore, the exogenous variables, which have an influence on the results, seem to contribute more in the latter forecasted hours than in the initial ones. And, in a similar way in both LSTM and GRU models.

Finally, it is worth mentioning that the trend over the forecasted hours seems to be affected by the parking sectors. Los Angeles follows a linear pattern (approximately 2.5\% and 0.5\% of RMSE increment each forecasted hour), whereas Wattens seems to be logarithmic, i.e. seems to be more stable in the latter hours (slightly increment of the error from 3 to 6 hours).

\section{Conclusions and Future Research}

In this paper, models from two RNN architectures (LSTM and GRU) have been developed for accurately forecasting parking availability in urban areas over a succeeding period of 6 hours. The results show that the forecasts of our models have lower error than previously proposed models (TDNN) for parking occupancy rate. A further development has been carried out by including exogenous variables like hourly weather and calendar effects. Furthermore, the different variables proposed have been intensively compared in order to analyze the suitability of whether or not to add these exogenous variables.

The results show that the GRU architecture achieves better results in nearly all cases compared to the LSTM and the TDNN versions. The obtained results for 1-hour forecasted case are promising with a RMSE of $4.5 \%$ and $4.4 \%$ for the parking sectors of Los Angeles and Wattens, respectively. Furthermore, it is important to note that, one of the objectives of this work is also to evaluate the behaviour of the forecasting models in a long-term way, six hours, and in this case, the best obtained results are RMSE of $17 \%$ and $11.1 \%$. Therefore, we consider that the developed models could be good candidates to be explored in order to use them in long-term parking guidance and information systems.

Moreover, when considering each of the exogenous variables one by one, it could be observed that day of the week improves the GRU models in more than $1.5 \%$ accuracy and the working day variable improves it in approximately $0.5 \%$. In contrast, the temperature variable presents an unexpected negative impact in the RMSE results, which can reach values of up to $4 \%$ more than with the endogenous option.

This work also provides insights about the inner mechanism of parking process through a data analysis. The insights gained from the analysis have been used in order to decide some of the modeling aspects. For example, it has been visually confirmed that the length of the sequences to use as input for the model is twenty-four. The analysis also demonstrates that spatial correlations arise between near sectors.

Future work could be focused on exploring how the developed forecasting models could be further improved incorporating spatial-temporal structured data as input. Parking sectors have linear dependencies in space and time with nearby sectors that can be exploited in order to improve the accuracy of the models. One way to use spatial information in NN is through the use of special architectures like GCNN (Bruna et al., 2013). 
Another interesting point is the use of transfer learning techniques that can help in the creation of models in new deployments of sensors, as historical data are scarce. This could be made between sectors that have similar behaviors.

Lastly, other exogenous variables could be investigated that can improve the accuracy of the models. Some of them could be social events and traffic flow in the areas surrounding the parking sectors.

\section{Acknowledgements}

Throughout this work, the authors have benefited from the support of the inLab FIB team at Universitat Politècnica de Catalunya and the company Worldsensing S.L.

\section{Data Availability}

The parking data used to support the findings of this study were supplied by Worldsensing S.L. under license and so cannot be made freely available. Requests for access to these data should be made to Jamie Arjona Martínez, email: jarjona@worldsensing.com.

The calendar and weather data used to support the findings of this study are available from the corresponding author upon request.

\section{Conflicts of Interest}

The authors declare that there is no conflict of interest regarding the publication of this paper.

\section{Funding Statement}

This research was funded by Secretaria d'Universitats i Recerca de la Generalitat de Catalunya [2017-SGR-1749] and under the Industrial Doctorate Program [2016-DI-79].

\section{References}

Bergstra, J. and Bengio, Y. (2012) 'Random Search for Hyper-Parameter Optimization Yoshua Bengio', Journal of Machine Learning Research, 13, pp. 281-305. doi: 10.1.1.306.4385.

Blythe, P. et al. (2015) 'Short-term forecasting of available parking space using wavelet neural network model', IET Intelligent Transport Systems, 9(2), pp. 202-209. doi: 10.1049/iet-its.2013.0184.

Caicedo, F., Blazquez, C. and Miranda, P. (2012) 'Prediction of parking space availability in real time', Expert Systems With Applications, 39, pp. 7281-7290. doi: 10.1016/j.eswa.2012.01.091.

Caruana, R. and Lawrence, S. (2001) 'Overfitting in Neural Nets: Backpropagation, Conjugate Gradient, and Early Stopping', in NIPS '00 Proceedings of the 13th International Conference on Neural Information Processing Systems, pp. 381-387. Available at: https://papers.nips.cc/paper/1895-overfitting-in-neural-nets-backpropagation-conjugate-gradient-and-early-stopping.pdf (Accessed: 26 March 2019).

Cho, K. et al. (2014) 'On the Properties of Neural Machine Translation: Encoder-Decoder Approaches', in Proceedings of SSST-8, Eighth Workshop on Syntax, Semantics and Structure in Statistical Translation, pp. 103-111. doi: 10.3115/v1/W14-4012.

Fu, R., Zhang, Z. and Li, L. (2017) 'Using LSTM and GRU neural network methods for traffic flow prediction', in Proceedings - 201631 st Youth Academic Annual Conference of Chinese Association of Automation, YAC 2016, pp. 324-328. doi: 10.1109/YAC.2016.7804912.

Glorot, X. and Bengio, Y. (2010) 'Understanding the difficulty of training deep feedforward neural networks', in Proceedings of the Thirteenth International Conference on Artificial Intelligence and Statistics, pp. 249-256. Available at: http://www.iro.umontreal. (Accessed: 26 March 2019).

Guo, C. and Berkhahn, F. (2016) Entity Embeddings of Categorical Variables. Available at: https://www.kaggle.com/c/rossmann-store-sales (Accessed: 22 March 2019).

Hochreiter, S. (1991) Fundamental Deep Learning Problem. Technische Universit“at Munchen. Available at: http://people.idsia.ch/ juergen/SeppHochreiter1991ThesisAdvisorSchmidhuber.pdf (Accessed: 19 February 2020).

Hochreiter, S. and Urgen Schmidhuber, J. (1997) 'Long short-term memory', Neural Computation, 9(8), pp. 1735-1780. Available at: http://www.bioinf.jku.at/publications/older/2604.pdf (Accessed: 21 May 2018).

Hsueh, E., Lu, -Chan and Liao, C.-H. (2018) A Parking Occupancy Prediction Approach Based on Spatial and Temporal Analysis, Intelligent Information and Database Systems. ACIIDS 2018. Lecture Notes in Computer Science, vol 10751. Springer, Cham. doi: 10.1007/978-3-319- 
75417-8_47.

Ishak, S., Kotha, P. and Alecsandru, C. (2003) 'Optimization of Dynamic Neural Network Performance for Short-Term Traffic Prediction', Transportation Research Record: Journal of the Transportation Research Board, 1836, pp. 45-56. doi: 10.3141/1836-07.

Jones, M. et al. (2018) 'ParkUs 2.0: Automated Cruise Detection for Parking Availability Inference'. doi: 10.1145/3144457.3144495.

Jozefowicz, R. and Zaremba, W. (2015) ‘An Empirical Exploration of Recurrent Network Architectures', in Volume 37: International Conference on Machine Learning. Available at: http://proceedings.mlr.press/v37/jozefowicz15.pdf (Accessed: 11 December 2018).

Kingma, D. P. and Lei Ba, J. (2014) ‘Adam: A Method for Stochastic Optimization.', in Published as a conference paper at the 3rd International Conference for Learning Representations 2015. San Diego. Available at: https://arxiv.org/pdf/1412.6980.pdf (Accessed: 26 March 2019).

Lin, T., Rivano, H. and Le Mouel, F. (2017) ‘A Survey of Smart Parking Solutions', IEEE Transactions on Intelligent Transportation Systems, 18(12), pp. 3229-3253. doi: 10.1109/TITS.2017.2685143.

Medeiros, M. C., Teräsvirta, T. and Rech, G. (2006) 'Building Neural Network Models for Time Series: A Statistical Approach'. doi: 10.1002/for.974.

Polson, N. G. and Sokolov, V. O. (2017) 'Deep learning for short-term traffic flow prediction', Transportation Research Part C: Emerging Technologies. doi: 10.1016/j.trc.2017.02.024.

Rajabioun, T. and Ioannou, P. (2015) 'On-Street and off-street parking availability prediction using multivariate spatiotemporal models', IEEE Transactions on Intelligent Transportation Systems, 16(5), pp. 2913-2924. doi: 10.1109/TITS.2015.2428705.

Srivastava, N. et al. (2014) 'Dropout: A Simple Way to Prevent Neural Networks from Overfitting', Journal of Machine Learning Research, 15, pp. 1929-1958. Available at: http://jmlr.org/papers/v15/srivastava14a.html (Accessed: 15 February 2020).

Teodorovic, D. and Lucic, P. (2006) 'Intelligent parking systems', European Journal of Operational Research, 175(3), pp. 1666-1681. doi: 10.1016/j.ejor.2005.02.033.

Vlahogianni, E. I. et al. (2014) 'Exploiting new sensor technologies for real-time parking prediction in urban areas', in TRB 93rd Annual Meeting Compendium of Papers, p. 19. Available at: https://pdfs.semanticscholar.org/056e/8de473e097a746dd9835ff1bf7cf375bd069.pdf (Accessed: 21 May 2018).

Vlahogianni, E. I. et al. (2016) 'A Real-Time Parking Prediction System for Smart Cities', Journal of Intelligent Transportation Systems, 20(2), pp. 192-204. doi: 10.1080/15472450.2015.1037955.

Vlahogianni, E. I. and Karlaftis, M. G. (2013) 'Testing and Comparing Neural Network and Statistical Approaches for Predicting Transportation Time Series', Transportation Research Record Journal of the Transportation Research Board Transportation Research Board of the National Academies, (2399), pp. 9-22. doi: 10.3141/2399-02.

Vlahogianni, E. I., Karlaftis, M. G. and Golias, J. C. (2005) 'Optimized and meta-optimized neural networks for short-term traffic flow prediction: A genetic approach', Transportation Research Part C: Emerging Technologies. doi: 10.1016/j.trc.2005.04.007.

Vlahogianni, E. and Karlaftis, M. (2011) 'Temporal aggregation in traffic data: implications for statistical characteristics and model choice', Transportation Letters, 3(1), pp. 37-49. doi: 10.3328/TL.2011.03.01.37-49.

Yanjie Duan, Yisheng Lv and Fei-Yue Wang (2016) 'Travel time prediction with LSTM neural network', in 2016 IEEE 19th International Conference on Intelligent Transportation Systems (ITSC). doi: 10.1109/ITSC.2016.7795686. 